\title{
CAPACITIES AND SPANS ON RIEMANN SURFACES ${ }^{1}$
}

\author{
JACOB BURBEA
}

\begin{abstract}
Let $K(z, z), R(z, z)$, and $C(z)$ be the values of the Bergman kernel, the reduced Bergman kernel and the analytic capacity on an open Riemann surface $\Omega$ (with respect to a local parameter $z$ ). Let $M(z)=$ $\pi K(z, z)$ and $S(z)=\sqrt{\pi R(z, z)}$. For $\Omega \notin O_{G}$ and for each integer $n>0$, it is shown that

$$
C^{(n+1)(n+2)}<(n+1) !\left(\prod_{k=0}^{n+1} k !\right)^{-2} \operatorname{det}\left\|M_{j k}\right\|_{j, k=0}^{n},
$$

where $C=C(z)$ and $M_{j \bar{k}}=\left(\partial^{j+k} / \partial z^{j} \partial \bar{z}^{k}\right) M(z)$. Equality occurs if and only if $\Omega$ is conformally equivalent to the unit disk less (possibly) a closed set of inner capacity zero. The special case of this result, namely when $n=0$, is due to Hejhal and Suita. Let $\kappa(z)$ be the curvature of the "span metric" $S(z)|d z|$. As an attempt to resolve a conjecture of Suita, we also show that for $\Omega \notin O_{A D}, \kappa(z)<-2$ for each $z \in \Omega$. Both results are proved by studying suitable extremal problems.
\end{abstract}

1. Introduction. Let $K(z, z), R(z, z)$, and $C(z)$ be the values of the Bergman kernel, the reduced Bergman kernel, and the analytic capacity on an open Riemann surface $\Omega$ (with respect to a local parameter $z$ ). We write $M(z)=$ $\pi K(z, z)$ and $S(z)=\sqrt{\pi R(z, z)}$. Following Schiffer [4], $S(z)$ will be called the span of $\Omega$ at $z$. A problem originated by Sario and Oikawa [3, p. 342], and followed by others, notably by Hejhal [1, p. 106] and Suita [5], is to find relations amongst the quantities $M(z), S(z)$, and $C(z)$. Concerning the relation between $M(z)$ and $C(z)$, Hejhal [1, p. 106] obtained an answer for finite Riemann surfaces $\Omega$ by showing that $M(z)>C^{2}(z)$ if $\Omega$ is not simply connected. The general case of this result was given by Suita [5]. He showed that for $\Omega \notin O_{G}, M(z) \geqslant C^{2}(z)$ with equality if and only if $\Omega$ is conformally equivalent to the unit disk less (possibly) a closed set of inner capacity zero. ${ }^{2}$

In the present paper we provide yet another generalization of Suita's result to include higher derivatives of $M(z)$ (compare [3, p. 114] and [2]). This result (Theorem 1), is proved by studying suitable extremal problems and employing in part a method of proof similar to that of Suita [5].

With regard to the span of $\Omega$, Suita [5], led by an earlier special result of

Received by the editors June 20, 1977 and, in revised form, August 20, 1978.

AMS (MOS) subject classifications (1970). Primary 30A31, 30A40, 30A44.

Key words and phrases. Bergman kernel, analytic capacity, span, curvature.

${ }^{1}$ Supported in part by NSF Grant MCS 7703449.

2 This equality statement is different than that of [5] where it has been stated erroneously that equality can occur when $\Omega \in O_{G}$. However, if $\Omega$ is a parabolic Riemann surface of positive genus, there always exists a nontrivial analytic differential with a finite norm (cf. [3, pp. 246-249]). Hence in this case $M(z)>0$ while $C(z)=0$. 
Zarankiewicz [6], has raised the following conjecture:

ConJeCture 1. Let $\Omega \notin O_{A D}$ and let $\kappa(z)$ be the curvature of $S(z)|d z|$. Then $\kappa(z) \leqslant-4$ and equality holds if and only if $\Omega$ is conformally equivalent to the unit disk less (possibly) a closed set expressed as a countable union of $N_{D}$ sets.

We were not able to resolve completely this conjecture. However, when $\Omega$ is an arbitrary plane region, we are able to show that $\kappa(z) \leqslant-2$ for all $z \in \Omega$ (Theorem 2). We also reformulate the above conjecture in, hopefully, a more manageable way (Conjecture 2).

2. A generalization. Let $\Omega \notin O_{G}$ and let $H_{2}(\Omega)$ be the space of square integrable analytic differentials on $\Omega$. In what follows there is no essential loss of generality in assuming global coordinates $z$ in $\Omega$. Therefore, for sake of simplicity, we assume that $H_{2}(\Omega)$ is in fact the space of square integrable analytic functions $f$ on $\Omega$. This is a Hilbert space normed by

$$
\|f\|=\left\{\iint_{\Omega}|f(z)|^{2} d x d y\right\}^{1 / 2} .
$$

Since $\Omega \notin O_{G}, H_{2}(\Omega)$ possesses a nonconstant Bergmen kernel function $K(z, \zeta), z, \zeta \in \Omega$.

Let $U_{z}$ be an open set around a fixed point $z \in \Omega$. We write

$$
A_{m}(z)=\left\{f \in C^{\infty}\left(U_{z}\right):\left(\partial^{k} / \partial z^{k}\right) f(z)=0, k=0,1, \ldots, m-1\right\},
$$

and

$$
S_{m}(z)=\left\{f \in H_{2}(\Omega):\|f\|^{2} \leqslant \pi\right\} \cap A_{m}(z), \quad m \geqslant 1 .
$$

We also let

$$
S_{0}(z)=\left\{f \in H_{2}(\Omega):\|f\|^{2} \leqslant \pi\right\}
$$

and note that $S_{0}(z)$ is in fact, independent of $z$. Clearly, $S_{m}(z)$, for any $m \geqslant 0$, is a nonempty closed convex subset of $H_{2}(\Omega)$.

For a fixed $\zeta \in \Omega$ and $m \geqslant 0$ we consider the following extremal problem

$$
\lambda_{m}(\zeta)=\max \left\{\left|f^{(m)}(\zeta)\right|^{2}: f \in S_{m}(\zeta)\right\} .
$$

This problem, of course, has (up to a rotation) a unique solution. Moreover, by a standard Hilbert space argument and a use of the reproducing property of $K(z, \zeta)$ one can show that

$$
\lambda_{m}(\zeta)=J_{m} / J_{m-1},
$$

where $J_{-1} \equiv 1$ and

$$
J_{m}=J_{m}(\zeta)=\operatorname{det}\left\|M_{j k}\right\|_{j, k=0}^{m}, \quad m \geqslant 0 .
$$

Here

$$
M_{j \bar{k}}=\frac{\partial^{j+k}}{\partial \zeta^{j} \partial \bar{\zeta}^{k}} M(\zeta), \quad M_{0 \overline{0}}=M(\zeta)=\pi K(\zeta, \zeta)
$$


As customary $H_{\infty}(\Omega)$ stands for the Banach space of bounded holomorphic functions $f$ on $\Omega$ normed by $\|f\|_{\infty}=\sup _{z \in \Omega}|f(z)|$. The analytic capacity is then given by

$$
C=C(\zeta)=\max \left\{\left|f^{\prime}(\zeta)\right|: f \in H_{\infty}(\Omega),\|f\|_{\infty} \leqslant 1, f(\zeta)=0\right\} .
$$

This maximum is uniquely attained (up to a rotation) by the Ahlfors function $F(z)=F(z: \zeta)$. Thus, $F(\zeta)=0$ and $F^{\prime}(\zeta)=C(\zeta)$.

Let

$$
h_{m}(z)=[h(z)]^{m}, \quad h(z)=\exp \left[-\left(G(z, \zeta)+i G^{*}(z, \zeta)\right)\right], \quad m \geqslant 0,
$$

be the multivalued function in $\Omega$, where $G(z, \zeta)$ is the Green's function of $\Omega$ and $G^{*}(z, \zeta)$ is its harmonic conjugate. Then $\left|h_{m}(z)\right|,\left|h_{m}^{\prime}(z)\right|$ and $h_{m}^{\prime}(z) / h_{m}(z)$ are single valued, and, moreover

$$
\left\|h_{m}^{\prime}\right\|^{2}=\iint_{\Omega}\left|h_{m}^{\prime}(z)\right|^{2} d x d y=m \pi, \quad m \geqslant 0 .
$$

We are now in a position to state our theorem. The special case when $n=0$ is due to Hejhal [1, p. 106] and Suita [5].

TheOREM 1. Let $\Omega \notin O_{G}$. For each integer $n \geqslant 0$ and $\zeta \in \Omega$ we have

$$
C^{(n+1)(n+2)} \leqslant(n+1) !\left(\prod_{k=0}^{n+1} k !\right)^{-2} \operatorname{det}\left\|M_{j k}\right\| \|_{j, k=0}^{n} .
$$

Equality holds if and only if $\Omega$ is conformally equivalent to the unit disk less (possibly) a closed set of inner capacity zero.

Proof. Let $\Omega \notin O_{G}$ and consider the function

$$
\phi_{m}(z)=\frac{1}{\sqrt{m+1}}[F(z)]^{m+1} \frac{h_{m+1}^{\prime}(z)}{h_{m+1}(z)}, \quad m \geqslant 0 .
$$

This function is analytic and single valued in $\Omega$. Also, since $F^{m+1} / h_{m+1}=$ $(F / h)^{m+1}$ is analytic in $\Omega,\left|F^{m+1} / h_{m+1}\right| \leqslant 1$. Therefore,

$$
\left\|\phi_{m}\right\|^{2}=\left\|\frac{F^{m+1}}{h_{m+1}} \frac{h_{m+1}^{\prime}}{\sqrt{m+1}}\right\|^{2} \leqslant\left\|\frac{h_{m+1}^{\prime}}{\sqrt{m+1}}\right\|^{2}=\pi .
$$

Further, by a direct computation, we obtain

$$
\phi_{m}^{(k)}(\zeta)=\sqrt{m+1} m ! C^{m+1} \delta_{k m}, \quad k=0,1, \ldots, m .
$$

Consequently, $\phi_{m} \in S_{m}(\zeta)$ and hence, using (2.1),

$$
\left|\phi_{m}^{(m)}(\zeta)\right|^{2}=(m+1)(m !)^{2} C^{2(m+1)} \leqslant \lambda_{m}(\zeta) .
$$

Therefore, according to (2.2),

$$
(m+1)(m !)^{2} C^{2(m+1)} \leqslant J_{m} / J_{m-1} .
$$

Upon multiplying the above inequalities, running from $m=0$ through $m=$ $n$, and, using (2.3), we obtain (2.4). Equality in (2.4) entails equality in (2.5) 
for each $m=0,1, \ldots, n$. This implies that $\left|F^{m+1} / h_{m+1}\right|=|F / h|^{m+1} \equiv 1$ or that $h$ is single valued. This holds, as in the proof of Suita [5], if and only if $\Omega$ is conformally equivalent to the unit disk less (possibly) a closed set of inner capacity zero. This concludes the proof of the theorem.

RemarKs. 1. It is easily verified that $C^{(n+1)(n+2)} / \operatorname{det}\left\|M_{j k}\right\|_{j, k=0}^{n}$ is an absolute conformally invariant function.

2. It is clear that inequality (2.4) is also valid for $\Omega \in O_{G}$. In this case a trivial equality occurs when $\Omega$ is planar. If $\Omega$ is a parabolic Riemann surface of positive genus then for $n=0$ we have a strict inequality namely, $M(z)>0$ $=C(z)$. For $n \geqslant 1$, however, there exist cases for which trivial equality in (2.4) occurs.

3. The curvature of the span. Let $\Omega \notin O_{A D}$ be a plane region, and denote by $D(\Omega)$ the class of all holomorphic functions $f$ in $\Omega$ for which the Dirichlet integral

$$
D[f]=\iint_{\Omega}\left|f^{\prime}(z)\right|^{2} d x d y
$$

does not exceed $\pi$. Then for $\zeta \in \Omega$ we have

$$
S(\zeta)=\max \left\{\left|f^{\prime}(\zeta)\right|: f \in \mathbf{D}(\Omega), f(\zeta)=0\right\} .
$$

The above maximum is uniquely attained (up to a rotation). We consider the conformally invariant metric $S(z)|d z|$. Its curvature is given by

$$
\kappa(z)=-S^{-2} \Delta \log S, \quad S=S(z),
$$

where $\Delta$ is the Laplacian operator. We note first the following straightforward lemma:

LEMMA 1. For $S=S(\zeta), \zeta \in \Omega$, we have

$$
2^{-1} S^{2} \Delta \log S=\max \left\{\left|f^{\prime \prime}(\zeta)\right|^{2}: f \in \mathbf{D}(\Omega), f(\zeta)=f^{\prime}(\zeta)=0\right\},
$$

and the maximum is uniquely attained (up to a rotation).

We first treat the finite case. For this purpose we let $C_{n}$ designate the class of all regular regions which are bounded by $n$ closed analytic disjoint curves. Thanks to the conformal invariance of the curvature we may assume, without loss of generality, that $\Omega \in C_{n}$. Moreover, for the same reason, we can also assume that $\zeta=\infty \in \Omega$ and that the area of the complement $E=\mathrm{C}-\Omega, m E$, is maximal amongst all regions $\Omega^{*}$ which are conformally equivalent to $\Omega$. Under these assumptions the closed analytic curves of $\partial \Omega$ are convex and $m E=\pi S^{2}(\infty)$ (cf. [4]).

Let

$$
p(z)=z+a / z+\cdots, q(z)=z+b / z+\cdots,
$$

be the horizontal and vertical slit functions, respectively, of $\Omega$. According to Schiffer [4] $p(z)+q(z)=2 z$ and 


$$
p(z)-q(z)=\frac{2}{\pi} \iint_{E} \frac{d u d v}{z-w}, \quad z \in \Omega, w=u+i v .
$$

It is well known that $a-b=2 S^{2}(\infty)$ and therefore,

$$
m E=\pi S^{2}(\infty)=\pi(a-b) / 2 .
$$

Also, $D[p-q]=2 \pi(a-b)$. We introduce the function

$$
\phi(z)=\frac{p(z)-q(z)}{\sqrt{2(a-b)}}, \quad z \in \Omega .
$$

Clearly, $\phi(\infty)=0$ and $D[\phi]=\pi$.

LEMMA 2. $|\phi(z)| \leqslant 1$ for all $z \in \Omega$.

Proof. Using a method of Ahlfors-Beurling (see [3, p. 177]) we can show that

$$
\left|\iint_{E} \frac{d u d v}{z-w}\right| \leqslant \sqrt{\pi m E}
$$

and the lemma then follows from (3.1) and (3.2).

We now define a second function

$$
\psi(z)=\frac{1}{2}[\phi(z)]^{2}, \quad z \in \Omega .
$$

$\psi$ maps $\Omega$ into a subset of $\{z:|z|<1\}$ and the convex closed analytic curves of $\partial \Omega$ are mapped onto curves of the same nature. For $n>1, \psi$ is not univalent but at most $2 n$-valent with $\psi(\infty)=\psi^{\prime}(\infty)=0$. Also, $\phi$ is at most $n$-valent [3, p. 142]. It is, therefore, very plausible (as indeed is the case when $n=1)$ that the total image area of $\Omega$ under $\phi^{2}$ is at most twice of that under $\phi$. That is, $D\left[\phi^{2}\right] \leqslant 2 D[\phi]=2 \pi$ or $D[\psi] \leqslant \pi / 2$. If this were true then it would be possible to resolve Conjecture 1 as the proof of Theorem 2 will show. We are led, therefore, to the following reformulation of Conjecture 1:

Conjecture 2. Let $\Omega \in C_{n}$ be a maximal region as before. Then $D[\psi] \leqslant$ $\pi / 2$ with equality holding if and only if $n=1$.

The case $n=2$ of the above conjecture is readily verified by the earlier work of Zarankiewicz [6]. Here, however, we prove the following:

TheOREM 2. Let $\Omega \notin O_{A D}$ be a plane region. Then $\kappa(\zeta) \leqslant-2$ for each $\zeta \in \Omega$.

Proof. We first show that $\kappa(\zeta) \leqslant-2$ when $\Omega \in C_{n}$ for any integer $n \geqslant 1$. Again, we may assume that $\Omega$ is maximal and $\zeta=\infty \in \Omega$. Let $\phi$ and $\psi$ be as before. According to Lemma 2,

$$
\begin{aligned}
D[\psi] & =\frac{1}{4} D\left[\phi^{2}\right]=\iint_{\Omega}|\phi(z)|^{2}\left|\phi^{\prime}(z)\right|^{2} d x d y \\
& \leqslant \iint_{\Omega}\left|\phi^{\prime}(z)\right|^{2} d x d y=D[\phi]=\pi .
\end{aligned}
$$


Therefore, $\psi \in \mathbf{D}(\Omega)$, and, since $\psi(\infty)=\psi^{\prime}(\infty)=0$, Lemma 1 shows that

$$
2^{-1} S^{2} \Delta \log S \geqslant\left|\psi^{\prime \prime}(\infty)\right|^{2}, \quad S=S(\infty) \text {. }
$$

But, using (3.2),

$$
\psi^{\prime \prime}(\infty)=\left[\phi^{\prime}(\infty)\right]^{2}=\frac{a-b}{2}=S^{2}
$$

and so $2 \leqslant S^{-2} \Delta \log S$ or $\kappa(\infty) \leqslant-2$. This proves the theorem when $\Omega \in C_{n}, n \geqslant 1$ is any integer. For the general case $\Omega \notin O_{A D}$ we let $\left\{\Omega_{m}\right\}$ be a canonical exhaustion of $\Omega$ such that $\partial \Omega_{m}$ consists of a finite number of analytic curves. Then $\kappa(\zeta)=\lim _{m \rightarrow \infty} \kappa_{\Omega_{m}}(\zeta)$ and since $\kappa_{\Omega_{m}}(\zeta) \leqslant-2$ for each $m$ the theorem follows.

\section{BIBLIOGRAPHY}

1. D. A. Hejhal, Theta functions, kernel functions, and Abelian integrals, Mem. Amer. Math. Soc., No. 129, 1972.

2. __ Some remarks on kernel functions and Abelian differentials, Arch. Rational Mech. Anal. 52 (1973), 199-204.

3. L. Sario and K. Oikawa, Capacity functions, Springer-Verlag, Berlin and New York, 1969.

4. M. Schiffer, The span of multiply connected domains, Duke Math. J. 10 (1943), 209-216.

5. N. Suita, Capacities and kernels on Riemann surfaces, Arch. Rational Mech. Anal. 46 (1972), 212-217.

6. K. Zarankiewicz, Über ein numerisches Verfahren zur konformen Abbildung zweifach zusammenhängender Gebiete, Z. Angew. Math. Mech. 14 (1934), 97-104. 15260

Department of Mathematics, University of Pittsburgh, Pittsburgh, Pennsylvania 